\title{
ON THE HYPER-SUMS OF POWERS OF INTEGERS
}

\author{
DIFFALAH LAISSAOUI, FOUAD BOUNEBIRAT, AND MOURAD RAHMANI
}

Received 27 May, 2016

\begin{abstract}
The main object of this paper is to study an old problem concerning the hyper-sums of powers of integers. First, we establish some important properties of this problem (generating function, explicit formula, congruence). Finally, an explicit formula for the hyper-sums of powers of integers involving the generalized Bernoulli polynomials are also given.
\end{abstract}

2010 Mathematics Subject Classification: 11B73; 11B68; 33C05

Keywords: Bernoulli numbers, sums of powers of integers, Stirling numbers

\section{INTRODUCTION}

Following the usual notations (see [4]), the falling factorial $x^{\underline{n}}(x \in \mathbb{C})$ is defined by $x^{\underline{0}}=1, x^{\underline{n}}=x(x-1) \cdots(x-n+1)$ for $n>0$ and, the rising factorial denoted by $x^{\bar{n}}$, is defined by $x^{\bar{n}}=x(x+1) \cdots(x+n-1)$ with $x^{\overline{0}}=1$. The (signed) Stirling numbers of the first kind $s(n, k)$ are the coefficients in the expansion

$$
x^{\underline{n}}=\sum_{k=0}^{n} s(n, k) x^{k} .
$$

The Stirling numbers of the second kind, denoted $\left\{\begin{array}{l}n \\ k\end{array}\right\}$ are the coefficients in the expansion

$$
x^{n}=\sum_{k=0}^{n}\left\{\begin{array}{l}
n \\
k
\end{array}\right\} x^{\underline{k}} .
$$

The Stirling numbers of the second kind $\left\{\begin{array}{l}n \\ k\end{array}\right\}$ count the number of ways to partition a set of $n$ elements into exactly $k$ nonempty subsets. 
The hyper-sums (sums of sums) of powers of integers $S_{p}^{(r)}(n)(p \geq 0)$ (or the $r$-fold summation of $p$ th powers) are defined recursively as

$$
\left\{\begin{array}{c}
S_{0}^{(r)}(n)=\left(\begin{array}{l}
n+r \\
r+1
\end{array}\right), r \geq 0 \\
S_{p}^{(0)}(n)=\sum_{i=1}^{n} i^{p} ; S_{p}^{(r)}(n)=\sum_{j=1}^{n} S_{p}^{(r-1)}(j), n, r, p \geq 1 .
\end{array}\right.
$$

As mentioned by Knuth [8], the hyper-sums of powers of integers was introduced for the first time by Faulhaber as a generalization of sums of powers of integers

$$
S_{p}^{(0)}(n)=1^{p}+2^{p}+\cdots+n^{p} .
$$

The sums $S_{p}^{(0)}(n)$ have been of interest for a long time and different methods have been proposed to obtain them, see $[1-3,5,6,9,10]$.

In 2005, Inaba [7] extended the well-known formulas for $S_{p}^{(0)}(n)$ which is based on Stirling numbers of the second kind to obtain the explicit formulas of the hypersums of powers of integers

$$
S_{p}^{(r)}(n)=\sum_{k=1}^{p} k !\left(\begin{array}{l}
n+r+1 \\
r+k+1
\end{array}\right)\left\{\begin{array}{l}
p \\
k
\end{array}\right\}, \quad n, p \geq 1 .
$$

Note that when $p \geq 0$, we can write $S_{p}^{(r)}(n)$ as

$$
S_{p}^{(r)}(n)=\sum_{k=0}^{p}\left\{\begin{array}{l}
p \\
k
\end{array}\right\}\left(k !\left(\begin{array}{l}
n+r+1 \\
k+r+1
\end{array}\right)-\left(\begin{array}{c}
n+r \\
r
\end{array}\right) \delta_{0, k}\right),
$$

where $\delta_{i, j}$ denotes the Kronecker symbol.

The purpose of the present paper is to give some results concerning the hyper-sums of powers of integers $S_{p}^{(r)}(n)$.

\section{MAIN RESULTS}

We begin by the exponential generating function of the hyper-sums of powers of integers.

Theorem 1. The exponential generating function of the hyper-sums of powers of integers $S_{p}^{(r)}(n)$ is

$$
\sum_{p \geq 0} S_{p}^{(r)}(n) \frac{z^{p}}{p !}=\left(\begin{array}{c}
n+r+1 \\
r+1
\end{array}\right){ }_{2} F_{1}\left(\begin{array}{c}
1,-n \\
r+2
\end{array} ; 1-e^{z}\right)-\left(\begin{array}{c}
n+r \\
r
\end{array}\right)
$$


where ${ }_{2} F_{1}\left(\begin{array}{c}a, b \\ c\end{array} ; z\right)$ denotes the Gaussian hypergeometric function defined by

$$
\sum_{n \geq 0} \frac{(a)^{\bar{n}}(b)^{\bar{n}}}{(c)^{\bar{n}}} \frac{z^{n}}{n !}
$$

Proof. First, we invert the formula (1.2) to obtain

$$
\sum_{k=0}^{p} s(p, k) S_{k}^{(r)}(n)=p !\left(\begin{array}{c}
n+r+1 \\
p+r+1
\end{array}\right)-\left(\begin{array}{c}
n+r \\
r
\end{array}\right) \delta_{0, p} .
$$

Further, we have

$$
\begin{aligned}
A_{0}(z) & =\sum_{p \geq 0}\left(p !\left(\begin{array}{c}
n+r+1 \\
p+r+1
\end{array}\right)-\left(\begin{array}{c}
n+r \\
r
\end{array}\right) \delta_{0, p}\right) \frac{z^{p}}{p !} \\
& =\sum_{p \geq 0}\left(\begin{array}{c}
n+r+1 \\
p+r+1
\end{array}\right) z^{p}-\left(\begin{array}{c}
n+r \\
r
\end{array}\right) \\
& =\left(\begin{array}{c}
n+r+1 \\
r+1
\end{array}\right) \sum_{p \geq 0} \frac{(1)^{\bar{p}}(-n)^{\bar{p}}}{(r+2)^{\bar{p}}} \frac{(-z)^{p}}{p !}-\left(\begin{array}{c}
n+r \\
r
\end{array}\right) .
\end{aligned}
$$

Finally, the result follows from [11, Theorem 3]

$$
\sum_{p \geq 0} S_{p}^{(r)}(n) \frac{z^{p}}{p !}=A_{0}\left(e^{z}-1\right) .
$$

This completes the proof.

We will now derive a few further consequences of Theorem 1 .

Corollary 1. The exponential generating function of the hyper-sums of powers of integers $S_{p}^{(r)}(n)$ is given by

$$
\sum_{p \geq 0} S_{p}^{(r)}(n) \frac{z^{p}}{p !}=\sum_{i=1}^{n}\left(\begin{array}{c}
n+r-i \\
r
\end{array}\right) e^{i z}
$$

Proof. It follows from the theory of hypergeometric functions that the Gaussian hypergeometric function ${ }_{2} F_{1}\left(\begin{array}{c}1,-n \\ r+2\end{array} ; 1-e^{z}\right)$ has an integral representation given by

$$
{ }_{2} F_{1}\left(\begin{array}{c}
1,-n \\
r+2
\end{array} ; 1-e^{z}\right)=(r+1) \int_{0}^{1}(1-x)^{r}\left(1-x+x e^{z}\right)^{n} d x
$$


Hence, we obtain

$$
\begin{aligned}
\sum_{p \geq 0} S_{p}^{(r)}(n) \frac{z^{p}}{p !} & =\frac{(n+r+1) !}{n ! r !} \sum_{k=0}^{n}\left(\begin{array}{l}
n \\
k
\end{array}\right) e^{(n-k) z} \int_{0}^{1}(1-x)^{r+k} x^{n-k} d x-\left(\begin{array}{c}
n+r \\
r
\end{array}\right) \\
& =\frac{(n+r+1) !}{n ! r !} \sum_{k=0}^{n}\left(\begin{array}{l}
n \\
k
\end{array}\right) e^{(n-k) z} \frac{(r+k) !(n-k) !}{(n+r+1) !}-\left(\begin{array}{c}
n+r \\
r
\end{array}\right) \\
& =\sum_{k=0}^{n}\left(\begin{array}{c}
k+r \\
r
\end{array}\right) e^{(n-k) z}-\left(\begin{array}{c}
n+r \\
r
\end{array}\right) \\
& =\sum_{k=0}^{n-1}\left(\begin{array}{c}
k+r \\
r
\end{array}\right) e^{(n-k) z},
\end{aligned}
$$

as claimed.

An explicit formula for $S_{p}^{(r)}(n)$ is given in the following corollary.

Corollary 2. The hyper-sums of powers of integers $S_{p}^{(r)}(n)(p \geq 0)$ is given by

$$
S_{p}^{(r)}(n)=\sum_{i=1}^{n}\left(\begin{array}{c}
n+r-i \\
r
\end{array}\right) i^{p} .
$$

Proof. Since

$$
\begin{aligned}
\sum_{p \geq 0} S_{p}^{(r)}(n) \frac{z^{p}}{p !} & =\sum_{i=1}^{n}\left(\begin{array}{c}
n+r-i \\
r
\end{array}\right) \sum_{p \geq 0} \frac{(i z)^{p}}{p !} \\
& =\sum_{p \geq 0}\left(\sum_{i=1}^{n}\left(\begin{array}{c}
n+r-i \\
r
\end{array}\right) i^{p}\right) \frac{z^{p}}{p !}
\end{aligned}
$$

Equating the coefficient of $\frac{z^{p}}{p !}$, we get the result.

In the next theorem we will present some congruence relations for $S_{p}^{(r)}(n)$.

Theorem 2. Let $n$ be a prime number and for $p>0, r \geq 0$, we have

(1) If $n \mid r+1$, then $S_{p}^{(r)}(n) \equiv 0(\bmod n)$,

(2) If $n \nmid r+1$ and $n-1 \mid p$, then $S_{p}^{(r)}(n) \equiv-1(\bmod n)$.

Proof. If $n \mid r+1$ then by Lucas' congruence we get

$$
\begin{aligned}
S_{p}^{(r)}(n) & \equiv \sum_{i=1}^{n-1}\left(\begin{array}{c}
r-i \\
r
\end{array}\right) i^{p}(\bmod n) \\
& \equiv 0(\bmod n) .
\end{aligned}
$$


Since $n-1 \mid p$, the Fermat's little theorem and Lucas' congruence gives

$$
\begin{aligned}
S_{p}^{(r)}(n) & \equiv \sum_{i=1}^{n-1}\left(\begin{array}{c}
n+r-i \\
r
\end{array}\right)(\bmod n) \\
& \equiv-1+\left(\begin{array}{c}
n+r \\
r+1
\end{array}\right)(\bmod n) \\
& \equiv-1(\bmod n) .
\end{aligned}
$$

This completes the proof.

Theorem 3. The ordinary generating function of the hyper-sums of powers of integers $S_{p}^{(r)}(n)$ is given by

$$
\sum_{r \geq 0} S_{p}^{(r)}(n) z^{r}=\frac{1}{(1-z)^{n+1}} \sum_{i=1}^{n}(1-z)^{i} i^{p}
$$

Proof. Since

which implies (2.2).

$$
\sum_{r \geq 0}\left(\begin{array}{c}
n+r-i \\
r
\end{array}\right) z^{r}=(1-z)^{i-n-1}
$$

Further, from (2.1) and (2.2), we deduce the double generating function of the hyper-sums of powers of integers $S_{p}^{(r)}(n)$

$$
\begin{aligned}
\sum_{r \geq 0} \sum_{p \geq 0} S_{p}^{(r)}(n) \frac{z^{p}}{p !} t^{r} & =\sum_{i=1}^{n} \sum_{r \geq 0}\left(\begin{array}{c}
n+r-i \\
r
\end{array}\right) t^{r} e^{i z} \\
& =\frac{1}{(1-t)^{n+1}} \sum_{i=1}^{n}\left(e^{z}(1-t)\right)^{i} \\
& =\frac{e^{(n+1) z}(1-t)^{n}-e^{z}}{(1-t)^{n}\left(e^{z}(1-t)-1\right)}
\end{aligned}
$$

Now, according to the well-known formula, for $n \in \mathbb{N}$ and $m \in \mathbb{N}^{*}$

$$
{ }_{2} F_{1}\left(\begin{array}{c}
-n, 1 \\
m
\end{array} ; z\right)=\frac{n !(z-1)^{m-2}}{(m)^{\bar{n}} z^{m-1}}\left[\sum_{k=0}^{m-2} \frac{(n+1)^{\bar{k}}}{k !}\left(\frac{z}{z-1}\right)^{k}-(1-z)^{n+1}\right],
$$

we can rewrite the exponential generating function of the hyper-sums of powers of integers $S_{p}^{(r)}(n)$ as

$$
\sum_{p \geq 0} S_{p}^{(r)}(n) \frac{z^{p}}{p !}=\frac{(-1)^{r} e^{r z}}{\left(1-e^{z}\right)^{r+1}}\left(\sum_{k=0}^{r}\left(\begin{array}{c}
n+k \\
k
\end{array}\right)\left(1-e^{-z}\right)^{k}-e^{(n+1) z}\right)
$$




$$
-\left(\begin{array}{c}
n+r \\
r
\end{array}\right)
$$

The next result gives an explicit formula for $S_{p}^{(r)}(n)$ involving the generalized Bernoulli polynomials. Recall that the generalized Bernoulli polynomials $\mathbb{B}_{n}^{(\alpha)}(x)$ of degree $n$ in $x$ are defined by the exponential generating function [12,13]

$$
\left(\frac{z}{e^{z}-1}\right)^{\alpha} e^{x z}=\sum_{n \geq 0} \mathbb{B}_{n}^{(\alpha)}(x) \frac{z^{n}}{n !}
$$

for arbitrary parameter $\alpha$.

In particular, $\mathbb{B}_{n}^{(1)}(x):=\mathbb{B}_{n}(x)$ denotes the classical Bernoulli polynomials with $\mathbb{B}_{1}(0)=-\frac{1}{2}$.

Theorem 4. For all $n, p, r \geq 0$, we have

$$
\begin{aligned}
& S_{p}^{(r)}(n)=\frac{p !}{(p+r+1) !} \mathbb{B}_{p+r+1}^{(r+1)}(n+r+1) \\
& \quad-p ! \sum_{j=0}^{r}\left(\begin{array}{c}
n+k \\
k
\end{array}\right) \frac{\mathbb{B}_{p+j+1}^{(j+1)}(j)}{(p+j+1) !}-\left(\begin{array}{c}
n+r \\
r
\end{array}\right) \delta_{0, p} .
\end{aligned}
$$

Proof. By (2.3) and (2.4) we have

$$
\begin{aligned}
\sum_{p \geq 0} S_{p}^{(r)}(n) \frac{z^{p}}{p !} & =-\sum_{k=0}^{r}\left(\begin{array}{c}
n+k \\
k
\end{array}\right) \frac{e^{(r-k) z}}{\left(e^{z}-1\right)^{r-k+1}}+\frac{e^{(r+n+1) z}}{\left(e^{z}-1\right)^{r+1}}-\left(\begin{array}{c}
n+r \\
r
\end{array}\right) \\
& =-\sum_{k=0}^{r}\left(\begin{array}{c}
n+k \\
k
\end{array}\right) \sum_{p \geq 0} \mathbb{B}_{p}^{(r-k+1)}(r-k) \frac{z^{p-r-1}}{p !} \\
& +\sum_{p \geq 0} \mathbb{B}_{p}^{(r+1)}(n+r+1) \frac{z^{p-r-1}}{p !}-\left(\begin{array}{c}
n+r \\
r
\end{array}\right) .
\end{aligned}
$$

After some rearrangement, we find

$$
\begin{aligned}
\sum_{p \geq 0} S_{p}^{(r)}(n) \frac{z^{p}}{p !}=\sum_{p \geq 0} \frac{z^{p}}{p !} & \left(p ! \frac{\mathbb{B}_{p+r+1}^{(r+1)}(n+r+1)}{(p+r+1) !}\right. \\
& \left.-\sum_{k=0}^{r} p ! \frac{\mathbb{B}_{p+r-k+1}^{(r-k+1)}(r-k)}{(p+r-k+1) !}\left(\begin{array}{c}
n+k \\
k
\end{array}\right)-\left(\begin{array}{c}
n+r \\
r
\end{array}\right) \delta_{0, p}\right) .
\end{aligned}
$$

Equating the coefficient of $\frac{z^{p}}{p !}$, we get the result. 
When $r=0$, Theorem 4 reduces to the known result

$$
S_{p}^{(0)}(n)=\frac{1}{p+1}\left(\mathbb{B}_{p+1}(n+1)-\mathbb{B}_{p+1}(0)\right)-\delta_{0, p} .
$$

\section{ACKNOWLEDGEMENT}

The authors would like to thank an anonymous reviewer for careful reading of this manuscript and their constructive remarks.

\section{REFERENCES}

[1] A. Beardon, "Sums of powers of integers." Am. Math. Mon., vol. 103, no. 3, pp. 201-213, 1996, doi: $10.2307 / 2975368$.

[2] R. Chapman, "Evaluating $\sum_{n=1}^{N}(a+n d)^{p}$ again," The Mathematical Gazette, vol. 92, pp. 92-94, 03 2008, doi: 10.1017/S0025557200182622.

[3] A. Edwards, "Sums of powers of integers: a little of the history." Math. Gaz., vol. 66, pp. 22-28, 1982, doi: $10.2307 / 3617302$.

[4] R. L. Graham, D. E. Knuth, and O. Patashnik, Concrete mathematics: a foundation for computer science. 2nd ed., 2nd ed. Amsterdam: Addison-Wesley Publishing Group, 1994.

[5] M. D. Hirschhorn, "Evaluating $\sum_{n=1}^{N}(a+n d)^{p}$," The Mathematical Gazette, vol. 90, pp. 114116, 03 2006, doi: 10.1017/S0025557200179197.

[6] F. Howard, "Sums of powers of integers via generating functions." Fibonacci Q., vol. 34, no. 3, pp. 244-256, 1996.

[7] Y. Inaba, "Hyper-sums of powers of integers and the Akiyama-Tanigawa matrix." J. Integer Seq., vol. 8, no. 2, pp. art. 05.2.7, 6, 2005

[8] D. Knuth, "Johann Faulhaber and sums of powers." Math. Comput., vol. 61, no. 203, pp. 277-294, 1993, doi: 10.2307/2152953.

[9] T. C. Kotiah, "Sums of powers of integers - a review." Int. J. Math. Educ. Sci. Technol., vol. 24, no. 6, pp. 863-874, 1993, doi: 10.1080/0020739930240611.

[10] I. Mező and J. L. Ramírez, "The linear algebra of the r-Whitney matrices." Integral Transforms Spec. Funct., vol. 26, no. 3, pp. 213-225, 2015, doi: 10.1080/10652469.2014.984180.

[11] M. Rahmani, "Generalized Stirling transform.” Miskolc Math. Notes, vol. 15, no. 2, pp. 677-690, 2014.

[12] H. M. Srivastava and J. Choi, Zeta and q-zeta functions and associated series and integrals. Amsterdam: Elsevier, 2012.

[13] H. Srivastava and P. G. Todorov, "An explicit formula for the generalized Bernoulli polynomials." J. Math. Anal. Appl., vol. 130, no. 2, pp. 509-513, 1988, doi: 10.1016/0022-247X(88)90326-5.

Authors' addresses

Diffalah Laissaoui

Dr Yahia Farès University of Médéa, Médéa 26000, Algeria

E-mail address: laissaoui.diffalah74@gmail.com

\section{Fouad Bounebirat}

University of Boumerdès, Department of Mathematics, Boumrdès 35000, Algeria

E-mail address: bounebiratfouadeyahoo.fr 
Mourad Rahmani

USTHB, Faculty of Mathematics, P. O. Box 32, El Alia 16111, Bab-Ezzouar, Algiers, Algeria

E-mail address: mrahmani@usthb.dz 